\title{
LAS COMPETENCIAS SOCIALES DE LOS INTELECTUALES TRANSFORMATIVOS ${ }^{1}$
}

\section{Resumen}

El presente ensayo da cuenta de una discusión que en los ámbitos escolar y educativo ha sido olvidada o dejada de lado, como es la que guarda relación con las competencias sociales que tiene y debe manejar el docente convirtiéndose así en un intelectual transformativo. Si bien en las discusiones escolares y educativas las competencias que deben adquirir los estudiantes son importantes, también lo es que la formación social que tenga el docente influye directamente en esas adquisiciones, coadyuvando a que el estudiante otorgue sentido a la realidad.

La discusión sobre las competencias sociales de los intelectuales transformativos se orienta desde la teoría educativa crítica, por cuanto éstas hacen un esfuerzo por ir más allá del orden social establecido a través de normas, valores, reglas e ideologías, entre otros, que resultan extrañas para los sujetos y mediante los cuales se ejerce el poder y la dominación sobre el conjunto de la población y en cambio, plantean la necesidad de desvelar las estructuras sociales con el fin de transformarlas, jugando en este proceso un papel fundamental los intelectuales que se hacen cargo de los procesos educativos y escolares a través de los cuales no sólo forman las nuevas generaciones, sino que se forman ellos mismos como intelectuales, comprometidos con las problemáticas sociales.

Palabras clave: Intelectuales, transformativos, competencias sociales, educación, escuela, sujeto, subjetividad.

\section{Abstract}

The present rehearsal gives bill of a discussion that in the environments scholar and educational it has been forgotten or side dropshot like it is it the one that keeps relationship with the social competitions that he/she has and it should manage the educational one becoming this way an intellectual transformative. Although in the

\footnotetext{
* Profesor Universidad Pedagógica Nacional, Bogotá.

1 Ponencia presentada en el XI Encuentro de Educación Internacional a Distancia. Universidad de Guadalajara (México). Diciembre 3-6 de 2002. Este ensayo tiene como base los planteamientos conceptuales de los teóricos de las pedagogías críticos, liderados por Henry Giroux. Se ha tomado como base fundamental el texto Los profesores como intelectuales, hacia una pedagogía crítica del aprendizaje, Paidós, Barcelona, 1990.

Texto recibido el 2 de abril de 2004 y aprobado el 18 de mayo de 2004.
} 
school and educational discussions the competitions that the students should acquire are important, it is also it that the social formation that has the educational one influences directly in those acquisitions cooperating to that the student grants sense to the reality.

The discussion on the social competitions of the intellectual transformatives is guided from the theory educational critic since this makes an effort to go beyond the established social order through norms, values, rules and ideologies among others that are strange for the fellows and through which it is exercised the power and the dominance on the population's group and on the other hand, it outlines the to keep awake necessity the social structures with the purpose of transforming them, playing in this process a fundamental paper the intellectuals that are taken charge of the educational and school processes through which don't only form the new generations but rather they are formed themselves as committed intellectuals with the social problems.

Key words: Intellectuals, transformatives, social competitions, education, school, fellow, subjectivity.

\section{Introducción}

El objetivo de este ensayo es plantear, desde una perspectiva pedagógica, cuáles podrían ser las competencias sociales que deben manejar los docentes para convertirse en intelectuales transformativos desde la postura de la teoría educativa crítica, en particular, y la teoría crítica en general. La postura pedagógica debe cruzar necesariamente por el convencimiento de los profesores sobre la acción que ejercen y el papel social que juegan, el cual no se limita únicamente a reproducir las condiciones sociales, sino que debe estar comprometido con la producción de esas condiciones; es decir, con la producción de sentido individual y social; siguiendo a Giroux, es la interiorización por parte del maestro de que son el tiempo (en sentido histórico y actual), el espacio, la actividad y el conocimiento, los elementos a partir de los cuales giran las dinámicas de las escuelas.

La realidad social cambió o mejor dicho la cambiaron hombres y mujeres, tanto académicos como sumergidos en el mundo de la cotidianidad. Al cambiar la realidad social, otros son los referentes con los que se vive hoy; los lenguajes, las tecnologías, así como las interacciones se han redefinido y resignificado. No basta con decir, toca adaptarnos a una sociedad en cons- tante cambio, compleja, caótica, sino que es necesario construir los referentes para que esos dichos y prácticas se concreticen y tanto personas como instituciones puedan desarrollar su subjetividad y su objeto social.

Los teóricos educativos críticos -liderados por Henry Giroux- han reactivado una discusión iniciada por Gramsci sobre los intelectuales, a los que este último llamó orgánicos y los primeros llaman transformativos, pero que en esencia se dirigen hacia lo mismo: la transformación de la sociedad. En concepto de ambos, esa transformación debe direccionarse desde posturas críticas que conduzcan al cambio de la realidad social. La postura crítica, en concepto de Haberlas, tiene dos postulados básicos como lo son desvelar y transformar (Vasco:1990), pero ¿qué se desvela y qué se transforma? En concepto de todos los críticos sociales, las estructuras opresoras de la sociedad (Mardones:1991), hoy día a esas estructuras sociales se le ha sumado un aspecto fundamental, como lo es la constitución de la subjetividad; ello se hace más evidente si se considera que las ciencias sociales no tienen sólo compromiso con la realidad social, como eje abstracto de las problemáticas que acontecen, sino también con los individuos; es decir, que las disciplinas no construyen sus objetos y sus métodos sólo con el ánimo de abordar unos saberes, sino con el objetivo de interactuar con las personas y la sociedades a las cuales van dirigidos esos saberes.

\section{La postura de los teóricos educativos críticos}

Desde esa perspectiva, el intelectual transformativo dirige su mirada hacia dos componentes fundamentales de la realidad; por un lado, el análisis de la sociedad en la cual desarrolla su acción y, por el otro, su contribución a ésta a través de su ejercicio pedagógico.

Para realizar este proceso de análisis, es necesario exponer las tesis centrales de Giroux, mediante las cuales sustenta sus planteamientos de Los profesores como intelectuales transformativos:

La teoría educativa crítica configura sus planteamientos examinando las tradiciones educativas que han contribuido a analizar el papel socializador de las escuelas; éstas son:

a) La visión estructural-funcional de la instrucción escolar.

b) El punto de vista fenomenológico, impulsado por la nueva sociología de la educación.

c) Una visión crítico radical, asociada con frecuencia al análisis neo- 
marxista de la teoría y la práctica educativas.

\subsection{La visión estructural- funcionalista: ¿cómo el orden social se alcanza y se mantiene?}

Para los teóricos educativos críticos, la visión estructural-funcionalista se preocupa por el problema de cómo se transmiten las normas y los valores sociales en el contexto de las escuelas. Este es un modelo sociológico que encuentra sus raíces en el positivismo y como tal dirige su mirada a la forma como las escuelas realizan un trabajo socializador que lleva a los estudiantes a la aceptación incuestionable de un conjunto de creencias, reglas y disposiciones, como elementos básicos para que la sociedad pueda funcionar. ¿Cómo el orden social se alcanza y se mantiene?; es la gran preocupación de esta sociología "el mérito de este enfoquen es triple: 1) aclara el hecho de que las escuelas no existen en un espléndido aislamiento, alejadas de los intereses de la sociedad en general; 2) detalla algunas normas específicas y propiedades estructurales del currículo oculto; 3) plantea cuestiones acerca del carácter específicamente histórico del significado y del control social en las escuelas" (Apple: 1977).
Aun teniendo a su favor los anteriores planteamientos, Giroux considera que esta visión no tiene en cuenta que el desarrollo se produce a partir del conflicto, acentuando la idea del consenso y la estabilidad más que la del movimiento; por ello no aparece en sus análisis, con fuerza el conflicto social ni los intereses socioeconómicos en pugna; ve a los estudiantes como receptores pasivos y como productos de la socialización; así mismo, no encuentra nada extraño en las creencias, valores y normas de las sociedades.

\subsection{El enfoque social fenomenológico: construyendo socialmente los significados}

Para los teóricos educativos críticos este enfoque concentra su interés en el postulado de que los significados son producto de las interacciones; por ello, consideran fundamentales las interacciones; del aula y los encuentros sociales; así, los significados son dados por las situaciones en las que participa el estudiante, los cuales son creados a partir de interacciones específicas. Profesores y estudiantes construyen socialmente los significados, proceso en el cual juega un gran papel lo histórico y evidencia que éstos no se limitan únicamente a existir en el mundo, lo que para esta visión pone en cuestión la dimensión objetiva del conocimiento.

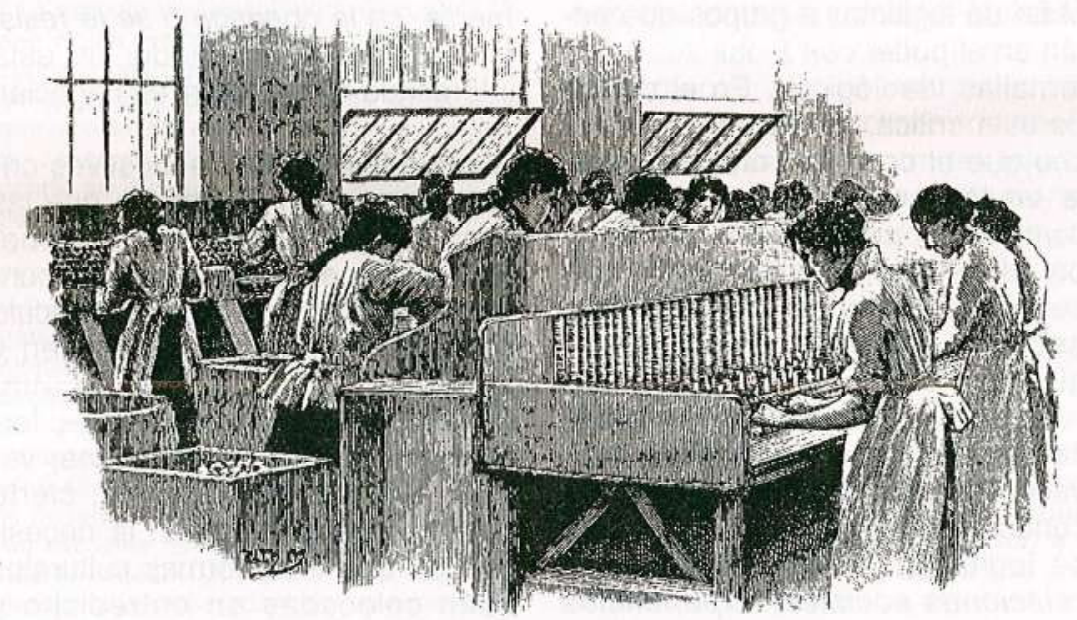

Para esta visión, es fundamental e hecho que los estudiantes participan en la definición y redefinición de sus mundos, desplazando el énfasis en la conducta institucional promulgada por el estructural-funcionalismo a las "interacciones con el lenguaje, las relaciones sociales y las categorías de significado". Para Giroux, los nuevos sociólogos educativos han señalado un punto clave al descubrir y analizar las relaciones entre socialización y currículo escolar, estableciendo la conexión entre la distribución de poder y el conocimiento: así, cuando oficialmente se seleccionan, organizan y distribuyen contenidos, estrategias, modelos, didácticas, se están presentando unas particulares visiones del mundo, de la ciencia, de las personas. En criterio de los seguidores de esta visión, el currículo no es inocente. Para contrarrestar estas posturas, es necesario que las personas que se encargan de elaborar los currículos de ciencias sociales, cuestionen los postulados que se pregonan tradicionalmente cuando se hace referencia a las bondades del currículo.

Giroux señala que la nueva sociología tiene también sus puntos débiles, la principal es que "representa una forma de idealismo subjetivo", en cuanto se centra en las interacciones, le da mucha relevancia a la intencionalidad de los estudiantes, pero no tiene teoría sobre el cambio y la conciencia sociales. Igualmente, señala Giroux y los teóricos educativos críticos, que no trabaja sobre el papel que juega la ideología en la construcción del conocimiento por parte de los estudiantes. Al ser un idealismo subjetivo dirige sus esfuerzos a la forma como los estudiantes perciben el mundo externo, pero sin hacer énfasis en que no siempre esa percepción se corresponde con la estructura y el contenido reales de ese mundo, el mundo no es sólo como lo percibimos individualmente, ni el individuo es sólo lo que se ve en el mundo. Para él el fracaso consiste en no poder esclarecer "de qué modos las es- 
tructuras sociales políticas enmascaran la realidad y promueven la hegemonía ideológica"(Gramsci, citado por Giroux:1990:69).

\subsection{La postura crítico-radical: relación entre reproducción económica y cultural}

La visión crítico radical sobre la socialización y el cambio social plantea la relación existente entre la reproducción económica y la cultural, por lo cual ve las escuelas como agentes de control ideológico "que reproducen y mantienen la vigencia de creencias, valores y normas dominantes" (p. 69), a partir de lo cual plantea que es necesario conectar las fuerzas macrosociales con microanálisis, estudios de aula, por ejemplo, a partir de los cuales se analice "cómo las condiciones sociales y económicas constriñen y distorsionan la construcción social de significado, particularmente en la medida en que dicha construcción ha sufrido la mediación del currículum oculto" (p. 70); así, a partir del proceso escolar se constata que existen unas estructuras sociales injustas que pueden ser identificadas y reemplazadas.

\subsection{Nuevos argumentos contra la instrumentalización de la escuela}

Giroux inicia sus planteamientos realizando una crítica a los planteamientos de la teoría educativa radical -en adelante ter-, argumentando que ésta se propuso la tarea de desvelar la forma como se producen la dominación y la opresión a partir de los mecanismos o dispositivos de la enseñanza escolar. Ello porque la ter se oponía a la idea de que las escuelas son los mejores lugares para promover y desarrollar la democracia, idea que estaba muy fortalecida en la década de los 70 y los 80 , a partir de la cual se afirmaba que haciendo democrática a la escuela se lograba la democratización de la sociedad; en criterio de esta teoría, esas posturas no tenían en cuenta que las escuelas promue-

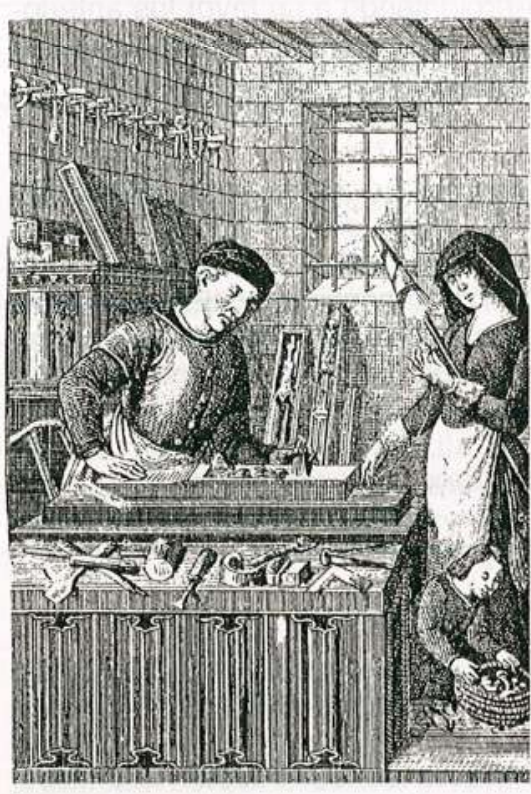

vitales y modos de razonamiento apropiado, y esta legitimación se da en una doble perspectiva: por un lado, legitimando a los estudiantes provenientes de las clases dominantes y por el otro, descalificando los lenguajes, relaciones sociales, las experiencias vitales y modos de razonamiento de los estudiantes de las clases trabajadoras.

La raíz de las posturas radicales no es compartida por Giroux, por considerar que éstas se atascan en el planteamiento de que las escuelas son únicamente lugares de reproducción social, a partir de la cual producen trabajadores obedientes para la lógica del capital, al igual porque perciben a los maestros atrapados en los procesos de dominación, ya que en concepto de los ter, el libreto ya está escrito y los maestros sólo pueden leerlo para actuarlo para cambiarlo; sería necesario cambiar las estructuras sociales porque en ellas radica la dominación e ideologización. Para Giroux, esta postura se convierte en un proceso inmovilizador por cuanto no permite que traspasen las contradicciones, luchas, rupturas y tensiones propias del proceso escolar y educativo y, en ese lenguaje de la dominación, se entierra la posibilidad de una pedagogía liberadora, impidiendo el diseño de estrategias educativas progresivas y con dimensión política, por cuanto de entrada niega la posibilidad de establecer mediaciones, pues con el dominador no se media, se le obedece o se le resiste; es más, el dominador no está interesado en mediar ni en negociar.

Aunque los teóricos educativos críticos comparten la idea de que las escuelas y la educación en general, establecen mecanismos de control teniendo como base el currículo mediante el cual se distribuyen y legitiman ciertas prácticas -lingüísticas, sociales, políticas-, formas culturales conocimientos, valores entre otros; es decir, cierto capital cultural, plantean la necesidad de que esas formas culturales sean colocadas en entredicho $y$ 
ampliadas a partir de la participación de otros individuos y grupos sociales, lo que hace necesario trabajar sobre los valores establecidos, porque en ellos se esconden las formas culturales a partir de las cuales se ejerce la dominación; reconociéndolas se puede luchar contra ellas y colocar en juego las propias.

Así las cosas, Giroux propone que, para que los planteamientos de los teóricos radicales se puedan llevar a cabo, es necesario que se combine el lenguaje de la crítica con el lenguaje de la posibilidad, a través de un discurso que supere el lenguaje fijado por las administraciones y el conformismo. La generación de ese nuevo discurso debe cruzar necesariamente por examinar y enjuiciar los defectos y fallos que hacen parte de la visión tradicional de la enseñanza escolar ${ }^{2}$ y por otra parte, "descubrir nuevas posibilidades de pensamiento y organización de las experiencias escolares" (p. 43).

La relación entre lenguaje y posibilidad se podrá establecer en la medida que se trabaje en dos frentes fundamentales: por un lado, la definición de las escuelas como esferas públicas democráticas (repensar el lenguaje de la instrucción escolar por cuanto en el ámbito político la preocupación por las escuelas no se dirige a cómo hacerlas más democráticas $^{3}$, sino cómo hacerlas

\footnotetext{
2 La escuela tradicional, para Giroux, se dirige a una racionalidad que tiene sus preocupaciones por la eficacia, los objetivos de conducta y los principios de aprendizaje que ven el conocimiento como un objeto de consumo y las escuelas como lugares donde se instruye a los niños que por alli pasan. En este sentido, imparte una cultura común.

${ }^{3}$ Las escuelas como esferas públicas democráticas se estructuran en torno a la investigación crítica, potenciando el diálogo significativo (que le diga algo a la gente en cuanto esté inscrito en sus experiencias y en su contexto) y la iniciativa humana (que la gente pueda hacer gala de la imaginación y no sea ajusticiada por ello ni académica ni materialmente). Como esferas públicas las escuelas no se reducen a las paredes que la rodean, simbólicamente hablando derrumba esas paredes para asociarse con
}

más eficaces y que contribuyan a la productividad económica). En este sentido, plantea que la fuerza ya no se coloca en aprender a leer críticamente el mundo, sino en que el estudiante domine los instrumentos de lectura; el dominio sobre los hechos se impone al dominio de la producción de sentido; y por el otro, la definición de los profesores como intelectuales transformativos (implementar pedagogías contrahegemónicas, a partir de las cuales se potencie al estudiante proporcionándole conocimientos y habilidades sociales necesarios para actuar en el conjunto de la sociedad, con sentido crítico y a la vez para la transformación).

Los profesores deben estar en capacidad de lograr que sean el tiempo, el espacio, la actividad y el conocimiento los elementos a partir de los cuales gire la dinámica de las escuelas; es decir, estructurar y desarrollar las condiciones para que como producto de la discusión y de la crítica, se vayan desechando las ideologías dominantes y dando lugar a espacios de confrontación de las mismas y nuevas posturas camino de su transformación. Los profesores como intelectuales transformativos deberán combinar la reflexión y la acción con el fin que, tanto ellos como los estudiantes, se conviertan en críticos que desvelan las estructuras de dominación con el objeto de su posterior transformación. Para ello, los profesores tienen que dotarse y dotar a los estudiantes de la caja de herramientas fundamental, la cual incluye las habilidades y los conocimientos necesarios para luchar contra las injusticias y la opresión y así mantener vivo el hecho histórico que habla de los diferentes vejámenes que han cometido quienes detentan el poder

las juntas de vecinos, las organizaciones barriales, la iglesia, la policía y así pensar la educación no sólo de los estudiantes que en un tiempo específico pasan por la escuela, sino del conjunto de la población que tiene posibilidades de ingresar a ella, proyectados en el espacio y en el tiempo. y a partir de los cuales han acallado las luchas; ello, mediante una memoria liberadora que posibilite el recuerdo, pero al igual lleve a la esperanza; no sólo se sufre la dominación, también se pueden producir procesos de resistencia a partir de los cuales se sueñe una vida, una sociedad, unas personas diferentes, y esos sueños se pueden conseguir a partir de pensar mutuamente la escuela, la educación, y la sociedad. El trabajo subjetivo que tendrá que realizar el docente será no convertirse en cómplice del orden social (García Canclini), teniendo presente ese orden como punto de partida de lo que quiere cambiar $y$ en el que se constituye como intelectual, pero al mismo tiempo analizando y realizando las transformaciones respectivas.

La comprensión por parte de los profesores de la necesidad de redefinir una política cultural con relación al resultado del conocimiento, es fundamental para el proyecto de teoría educativa crítica. La pedagogía debe entenderse como un conjunto concreto de prácticas que llevan a determina-

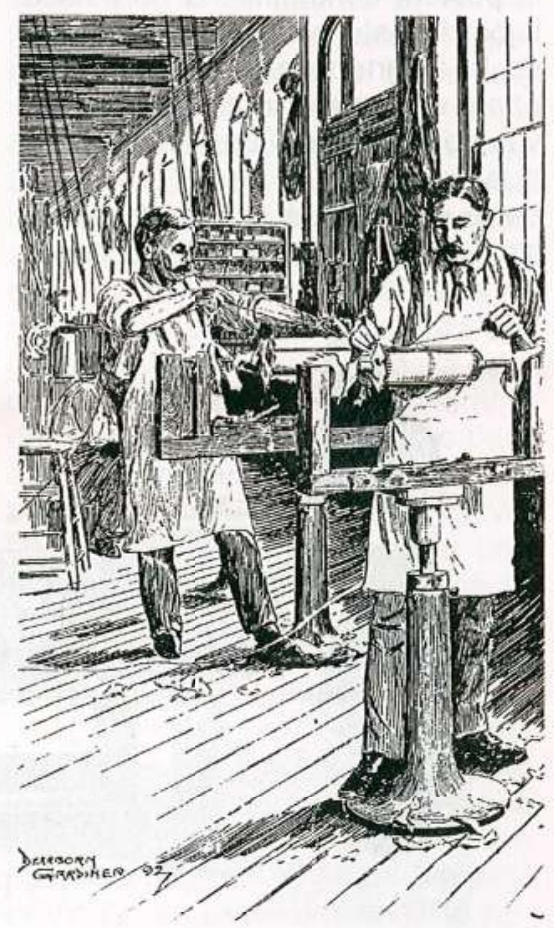


das formas sociales, conocimientos, experiencias y subjetividades específicas, de lo cual se desprende que la escuela puede moldear la forma de ser y de constitución en forma perversa o crítica, sin que el individuo intervenga o mediante su participación, con un proyecto democrático o autoritario, con contenidos o núcleos problémicos. En todos los casos, participa el individuo, haciendo, dejando hacer o las dos cosas. Es necesario tener claro que todos los proyectos encarnan intereses particulares, por lo tanto, desde la postura crítica, se trata de estar al tanto tratando de desvelar los intereses y el sentido de los demás y colocar sobre la mesa los propios.

\section{Intencionalidades de la formación pedagógica}

En el centro del proceso educativo subyace la idea de que las personas se formen para responder a las exigencias sociales y en este sentido, las posturas críticas consideran que la educación no puede hacerlo todo, pero tampoco es posible que no haga nada (Paulo Freire); la postura durkheimiana no estaba lejos de este planteamiento cuando le asignaba a la educación el papel de socializadora de las nuevas generaciones; Bruner le asigna a la educación el papel de puerta de la cultura; los sabios de la misión de ciencia y tecnología consi-

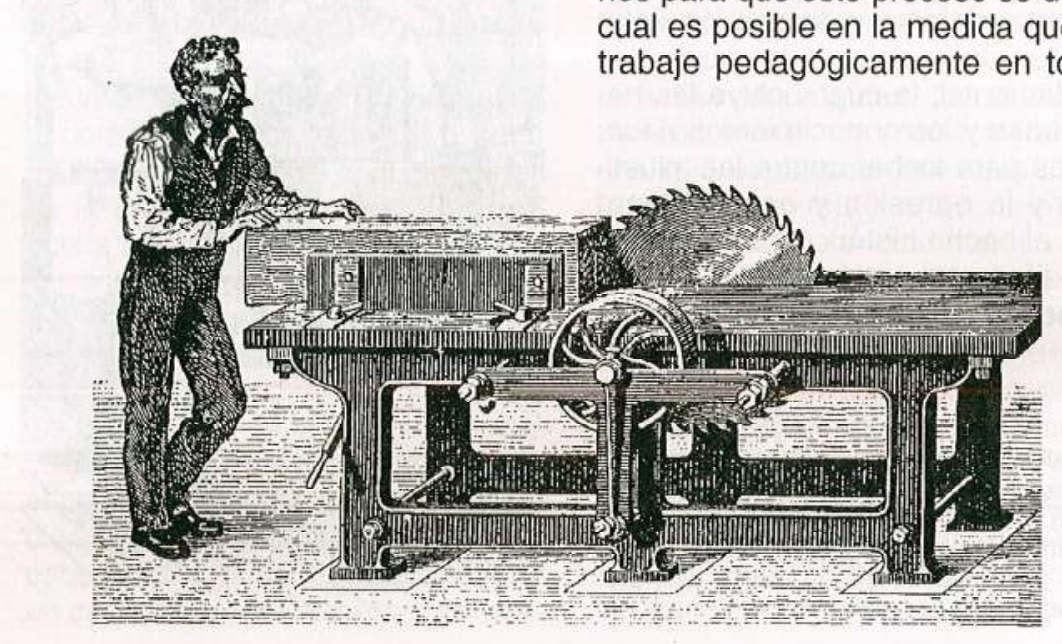

deran que sólo mediante la educación, Colombia logrará salir del precario estado social en el que se encuentra; para la Unesco, la educación encierra un tesoro y así se podría seguir nombrando las premisas que, puestas sobre la mesa, se convierten casi en etiquetas; sin embargo, el problema de fondo es que, en los diferentes ámbitos de la vida social se etiquetaron esas premisas y se trata a la educación como un bien de consumo y no de formación y desarrollo. De igual forma, poco se hace en pos de concebirla como un bien público y más bien se habla de ella como gasto social.

Detrás de la problemática se encuentra el hecho de que, a pesar de existir suficiente investigación y literatura sobre la importancia de la educación para el desarrollo social, ésta se ha instrumentalizado, respondiendo más a la lógica del capitalismo que a la de la democracia, y ello porque no se ha formado a los docentes con competencias sociales mediante las cuales sobresalga en el hecho educativo la formación de los sujetos que van producir la democracia y se ha colocado el énfasis en las personas que van a reproducir los hechos sociales. Aunque es claro que la educación no sólo se limita a reproducir las condiciones sociales, también lo es que para que ello sea posible es necesario construir las condiciones para que este proceso se dé, lo cual es posible en la medida que se trabaje pedagógicamente en torno a la dominación e ideologización que desarrollan quienes detentan el poder a través de los procesos escolares (diseño del currículo, aplicación y evaluación). Ahora mismo, en la escuela se trabaja con proyectos educativos institucionales, los cuales manejan una fundamentación de apertura democrática en cuanto sostienen que a través de ellos se va a construir la institución que quieren los equipos docentes y las instituciones específicas desde las necesidades sociales; sin embargo, un acercamiento -no sistemático todavía- a los PEI permite ver que éstos, en muchos casos se convierten en trozos de papel que les dice muy poco a los que viven la institución en el día a día ${ }^{4}$.

${ }^{4}$ Las competencias en el ámbito educativo. Cuando en los procesos educativos se hace referencia a las competencias generalmente enfocamos la mirada analítica a dos aspectos básicos: por un lado, a los conocimientos y capacidades genéricos que debe quedarle al estudiante para desenvolverse en diferentes situaciones sin que tenga que recurrir al libro, al experto, a lo dado, a la memoria y más bien estas competencias se dirijan a las pistas cognoscitivas en el orden teórico y práctico para saber hacer en contexto. Por el otro, al conjunto de conocimientos que la escuela debe proveer en el curso de su acción educativa. En ambos casos, las competencias sociales quedan relegadas a un segúndo plano, es decir, aunque los Proyectos Educativos Institucionales se refieran a ellas bien sea en sus énfasis, ejes o ambientes en la cotidianidad son desplazadas en aras de los contenidos. En otras palabras, la discusión en torno a las competencias gira en torno a un círculo vicioso, lo que debe ser capaz de hacer el estudiante, dirigiendo la mirada en la práctica a los contenidos en forma de logros, indicadores, aprendizajes, conocimientos y se deja de lado o se coloca poco énfasis en la formación social del estudiante y en la otra arista del problema: las competencias sociales que debe manejar el intelectual transformativo.

Lo que quiero argumentar es que en ese proceso de formar a los estudiantes con competencias sociales, es muy importante la postura asumida por los docentes y las propias competencias sociales que estos últimos tengan para llevar a cabo ese proceso, es decir, la capacidad de ser intelectuales transformativos: ¿cómo son vistas las competencias sociales desde la postura de la teoría educativa crítica? ¿Cuáles son los principales componentes de sus planteamientos? 


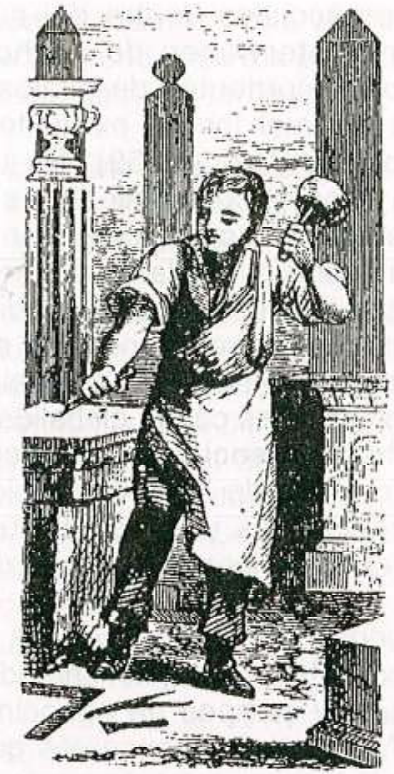

Igualmente, existe en esos PEI, una visión tradicional de las competencias, sobresaliendo en su definición estereotipos culturales, representaciones sociales, posturas ideológicas, lógicas encontradas, entre otras, que tienen estrecha relación con las concepciones y relaciones de clase, etnia y género mediante las cuales se implementan determinados tipos de conocimiento de acuerdo a esas concepciones y relaciones: los currículos de las clases dominantes son diferentes a los de las clases bajas, pero sobre todo, circulan a través de formas culturales que los legitiman y los convierten en representaciones sociales, convirtiéndose en procesos de extrañamiento para las clases trabajadoras, mediante los cuales los discursos y las prácticas se ven alejados de su constitución subjetiva y procesos sociales.

Asi, la mayoría de padres de familia de la clase baja y media le piden y le exigen a la escuela que le enseñe a sus hijos a leer, escribir, las operaciones básicas y algunos modales básicos; con esto se dan por bien servidos. Lo paradójico es que los docentes también piensan así. En esta forma de ver las competencias, sobresale el esquema de vuelta a lo básico, enseñar lo mínimo siempre y cuando sea fundamental para que el estudiante dé cuenta de su proceso normativo. Desde luego, lo básico no es definido ni por el docente, ni por el estudiante, sino por los organismos internacionales; el Ministerio de Educación Nacional, por su parte, hace creer que fueron sus expertos quienes lo definieron; por lo tanto, es necesario que los docentes repiensen el lenguaje y las prácticas implementadas en la escuela y que se examinen críticamente las propias posturas ideológicas, las representaciones sociales, los estereotipos, las actitudes que hacen parte de su propio lenguaje, teniendo en cuenta que dicho lenguaje conduce a ayudar a estructurar unas experiencias específicas, además, que esas posturas ideológicas son las que constituyen el marco teórico con el que los profesores se desenvuelven en el mundo académico y cotidiano, priorizando una parte del mundo y dejando por fuera otras; lo que está al alcance y lo que está fuera del alcance.

La democracia, por ejemplo, se enseña como un sistema político que guarda relación con elecciones, leyes, presidentes, ministros... muy poco se dice y se escribe en los discursos y prácticas educativas sobre los saqueos, las componendas, la compra de votos municipales, la compra de conciencias para votar un proyecto, eso le importa muy poco a los docentes y más bien se presenta la democracia como un proceso transparente, en constante evolución, sin baches, sin vericuetos, como el estado ideal. al cual, a pesar de las dificultades actuales, algún día llegaremos; por lo tanto, no se trabaja la pedagogía como hecho político, social y cultural ( $\mathrm{Pe}$ ña: 2000), dejando de lado los aspectos simbólicos de la democracia mediante los cuales en el futuro se podrían producir las resistencias por parte de los sujetos y los grupos sociales. Los currículos no se complejizan, sino que se presentan como un mundo ideal, sin conflictos, marcos teóricos que conciben al mundo de la misma forma, las ciencias y los problemas sociales no son problematizados, la demo cracia es vista y trabajada en las escuelas como un problema curricular y no como un problema del mundo cotidiano. Por ello, la insistencia de Giroux de situar las escuelas dentro de un contexto sociopolítico, esto es, quitar el énfasis en los contenidos para situar la mirada en "¿cómo determinados aspectos de la cultura colectiva se presentan en la escuela como conocimientos objetivos, fácticos?... ¿cómo consigue el conocimiento oficial representar las configuraciones ideológicas de los intereses dominantes en una sociedad? ¿cómo legitiman de hecho las escuelas estos patrones limitados y parciales de conocimiento como verdades incuestionadas?". En este pensamiento confluyen teóricos como Bourdieu, Bernstein, Apple y Giroux, entre otros.

En los currículos de los grupos dominantes se encuentran énfasis, discursos y prácticas y toda una gama simbólica, diferentes a los de los las clases trabajadoras; a los estudiantes se les inculca -proceso en el que los padres de familia juegan un papel fundamental-su papel de futuros líderes y dirigentes, direccionando los PEI por ese camino, lo cual denota que el problema de la formación y desarrollo social radica en el diseño e implementación de una política cultural, que para el caso de las clases dominantes sí existe; lograr que exista para las otras clases, es una tarea de la educación y la pedagogía en general, y del maestro en particular, pero para ello es necesario que éste se convierta en intelectual transformativo, su tarea consiste en generar una autoridad emancipadora a través de un trabajo intelectual y dialéctico, con compromiso social, ayudando al estudiante a formarse mediante un conocimiento crítico de la realidad social, creando una política de la verdad que defina la verdad como aquello que libera; es decir, la implementación por parte del profesor de una pedagogía crítica. Esta pe- 
dagogía consiste en reconocer la subjetividad (Touraine:1997) como la posibilidad de seguir diferentes modelos de identidad cultural -postulado contrario al de la modernidadmediante un compromiso social y político de carácter verdaderamente democrático, guiado por la reflexión y la acción.

\subsection{La formación de los docentes en las facultades de educación}

En el centro del problema de los intelectuales transformativos subyace la formación que reciben los docentes en las facultades de educación, la cual está orientada más por la profesionalización que por la disciplinarización (CNA:1998). En este sentido, los currículos están más orientados por la idea o ideas en torno al perfil profesional y ocupacional que por el sentido epistemológico (Canguilhem:1998) social y cultural que tienen las disciplinas, dejando de lado el componente de transformación que pueden producir los disciplinados 5 .

Por ello, es necesaria una visión curricular que contemple la finalidad democrática y pedagógica de las escuelas y, en este sentido, dirija sus acciones hacia la liberación; es decir, a que los individuos se constituyan como sujetos en el marco de la sociedad en la cual se encuentran inmersos.

La teoria educativa crítica es consciente de que la escuela legitima -a través de los currículos, discursos.y prácticas- ciertas formas de conocimiento e intereses culturales; sin embargo, pretende que esas formas sean cada vez más el producto de las apropiaciones culturales; es decir, que la gente lea críticamente la realidad y estando inmersa en los procesos socioculturales in-

\footnotetext{
${ }^{5}$ Este término hace mención al representante epistemológico de una disciplina, mediante el cual se piensa a sí mismo como posibilitador critico de cambios en la sociedad.
}

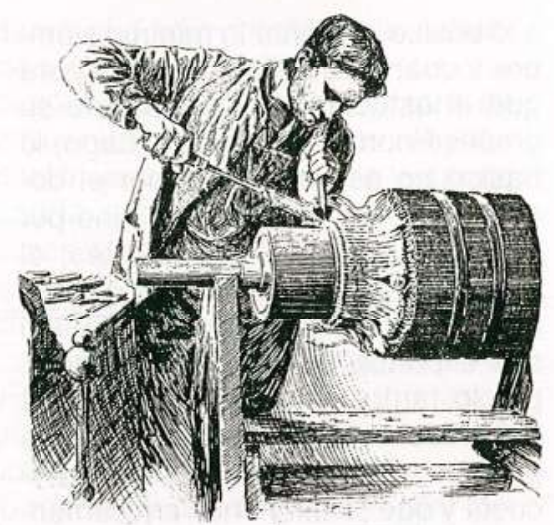

tervenga en el curso de la historia, haciendo incluir su cultura, sus formas culturales en los procesos escolares y sociales. Esto último, se ha venido dando en una forma aún no legitimada a través de la dinámica del currículo oculto, mediante la cual estudiantes y docentes se resisten a las dinámicas tradicionales de las escuelas.

Giroux y Apple, consideran que en lugar de pensar el curriculum como un proceso que genere comprensión interpretativa y aprendizaje intencional, es necesario un nivel crítico que lo direccione como estudio de la ideología en cuanto detrás de las propuestas curriculares se encuentran inmersos procesos de dominación y control y por lo tanto se trata de desvelarlas y transformarlas. Para ellos, basta con revisar críticamente algunas preguntas para darse cuenta de esta situación: "¿qué conocimientos entran a formar parte del curriculum?, ¿cómo se producen esos conocimientos?, ¿cómo se transmiten esos conocimientos en el aula?, ¿qué tipos de relaciones sociales del aula sirven para establecer un parangón y reproducir los valores y normas incorporados en las relaciones sociales aceptadas de otros ámbitos sociales dominantes?, ¿quien tiene acceso a formas legítimas de conocimiento?, ¿a qué intereses sirve este conocimiento?, ¿cuáles son las contradicciones y tensiones sociales y políticas mediatizadas a través de formas aceptables de conocimiento y rela- ciones sociales dentro del aula?, ¿cómo intervienen de hecho los métodos corrientes de evaluación para legitimar formas existentes de conocimiento?" (p. 59). En tanto para ellos el conocimiento es una construcción social, proponen que los modelos curriculares desarrollen formas de comprensión mediante las cuales se relacionen las explicaciones que se le dan a los significados sociales con la globalidad de los hechos sociales; es decir, la interrelación de los niveles micro y macrosociales y así emitir juicios sobre las pretensiones de verdad.

La pedagogía como disciplina busca constituir su objeto, método de estudio y campos de conocimiento. Así como los conceptos que la atraviesan como tal, "en este contexto, han mediado tanto intereses por: a) establecer la legitimidad epistemológica de este campo de estudio al intentar definir su estatuto paradigmático de disciplina, b) definir su carácter mediático en función de la transmisión social y cultural de órdenes simbólicos dominantes o, c) favorecer los problemas específicos de su heterogeneidad contextual tales como procesos comunicativos, actores, situaciones, relaciones sociales, modelos pedagógicos, innovaciones, etc." (Díaz: 1990: 39-49). Ello justifica involucrarla en los planteamientos, camino del profesor como intelectual transformativo, por cuanto ella es la encargada de reconceptualizar, conceptualizar y aplicar los saberes que circulan socialmente y darles proyección social y política; en este sentido, su acción se dirige por dos caminos: por un lado, va tras la intencionalidad de lo que se hace y pretende hacer educativamente, no sólo con el ánimo de sacarlo a la luz, sino de ser propositiva, y por el otro, basada en el saber que ha ido construyendo, propone modelos, innovaciones, estrategias, didácticas, enfoques, técnicas que potencien el sistema educativo. Por ello, es fundamental la vigilancia que se debe establecer frente a las competencias, porque están resultando 
más de 10 mismo y sólo mediante una actitud crítica de los profesores se puede cambiar la historia.

\subsection{Las categorías pedagógicas de los intelectuales transformativos}

Que el maestro se piense como intelectual tiene que ver con la visión tecnocrática de la educación, visión que percibe a esta última como un instrumento para el desarrollo y el cambio y, por lo tanto, que contemple la finalidad democrática y pedagógica de las escuelas y en este sentido dirija sus acciones hacia la liberación; es decir, a que los individuos se constituyan como sujetos en el marco de la sociedad en la cual se encuentran inmersos.

Se establece entonces, que un docente como intelectual transformativo es garante de los procesos y dinámicas que promueve en sus estudiantes, dando cuenta en sí mismo y por sí mismo de competencias sociales, lo cual le permite promover en el aula una estrecha relación entre sus procesos y los de los estudiantes. Este intelectual, tiene como referente de sus acciones el currículo con el cual se resiste y es alternativo a los ejercicios de poder de las instituciones estatales. Para ello, es necesario incorporar lo que Giroux Ilama una nueva sociologia del curriculum que, entre otros aspectos, contemple:

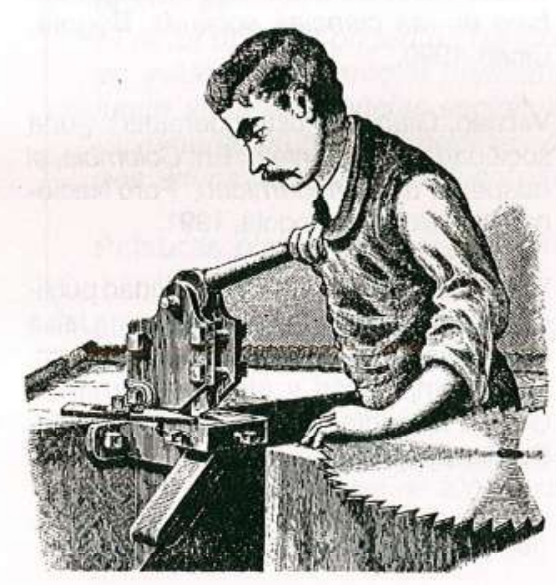

* Articular el hecho educativo con el contexto histórico en el cual se desarrollan los hechos. Ello permite hacer procesos comprehensivos según el momento histórico y desde allí analizar los hechos sociales, incorporando a la acción su función ética como docente (trabajar sobre los valores establecidos con miras a analizarlos y transformarlos si es necesario) y su función política (se teoriza críticamente para unos individuos y unos grupos sociales específicos con miras a resolver problemas sociales).

* Interactuar escolar y educativamente a partir de diferentes teorías del cambio y la conciencia sociales.

* Trabajar en torno al papel que juega la ideología en los procesos de construcción de conocimiento. En este sentido, problematizar el postulado crítico radical que ve "las escuelas como agentes de control ideológico".

* Problematizar la forma como las escuelas realizan un trabajo socializador.

* Combinar teorías, paradigmas, corrientes, escuelas, enfoques y conceptos, entre otros, que combinen el lenguaje de la crítica con el lenguaje de la posibilidad.

* Implementar pedagogías contrahegemónicas.

* Pensar el currículo desde una perspectiva democrática.

Ese cambio histórico, camino de la transformación del docente en intelectual transformativo, cruza necesariamente por la incorporación de tres categorías básicas; esto es, la interdisciplinariedad, la comunicación y la relación pedagógica.

La interdisciplinariedad, en cuanto es fundamental la incorporación de discursos y prácticas que tradicionalmente han estado vedados por los círculos de poder, más si se tiene en cuenta lo que se ha sostenido en este ensayo, como lo es la ampliación de las formas culturales, conocimientos, prácticas, representaciones sociales, etc.; en últimas, se trata de no tratar los hechos y las relaciones sociales como cosas.

La comunicación, por cuanto en un mundo tan incierto como el actual, se convierte en un dispositivo necesario para que fluyan los puntos de vista, las posturas, las conceptualizaciones, las categorizaciones $y$, desde luego, las neurosis, las incomprensiones, las rupturas, la luchas, etc. Es decir, tener la comunicación como punto de partida no asegura la desaparición de las luchas socioculturales - lo cual tampoco es necesario- pero sí abre el camino hacia el entendimiento entre los sujetos, las negociaciones, las mediaciones; es decir, hacia el intercambio de subjetividades.

La relación pedagógica debe permitir que en las aulas circulen culturas y con ellas la diversidad y la pluralidad; es decir, las relaciones de clase, las etnias, los géneros, porque sólo ello permitirá constituir y construir relaciones democráticas en la medida que se coloquen en juego las formas culturales, las de todos, no sólo las de unos pocos.

\section{Conclusiones}

La educación del presente siglo tiene las condiciones para convertirse en el lenguaje de la posibilidad; nunca antes se había visto expuesta a tantas presiones y se le había exigido tanto. Sin embargo, es necesario que las personas que se ocupan de la educación tengan presente que la acción de ésta es, ante todo, de proyección social, que compromete el futuro de los que pasan por las escuelas y de la sociedad. Por lo tanto, hay que estar cualificándola día a día. En este camino y como la señala Giroux, la educación se encuentra inscrita dentro de los estudios sociales, como tal se ve abo- 
cada a dos tareas fundamentales: por un lado, "identificar los procesos sociales que actúan en contra de la intención ética y política de la enseñanza escolar en una sociedad democrática y, por otro, poner a punto nuevos elementos que sirvan de apoyo a programas encaminados a la renovación de los estudios sociales" (p. 65). Así, una tarea fundamental es poder señalar las contradicciones entre el curriculum oficial y el curriculum oculto; es decir, entre los objetivos explícitos que la escuela y el sistema educativo en su conjunto muestran y desarrollan, con las normas, valores, creencias no explícitas que se transmiten y se viven por los estudiantes y que se convierten en parte fundamental de la vida escolar.

¿Qué mejor manera de transformarla que a través del compromiso del docente con la acción social que ejerce? Es claro que los profesores en su conjunto, no se piensan a sí mismos como intelectuales transformativos, sino que en su mayoría ejercen su trabajo de forma individual y mecánica, preocupándose más por acabar el programa que por influir en la conciencia de los estudiantes que tienen a su cargo; desde luego, hay que reconocer que se tienen grandes presiones de parte de las autoridades educativas y de los grupos que ostentan el poder, pero aun así, el poder de cambio del estado de cosas es enorme porque el docente trabaja con el conocimiento y éste hace parte de las transformaciones producidas en la última parte del siglo $X X$.

De esta forma, las competencias sociales del profesor son fundamentales para las luchas correspondientes, en tanto ellas conllevan nuevas formas de ver la realidad y, sobre todo, concretiza el cambio social, ya que lo ubica en personas específicas; esas competencias sociales deben dirigirse, sobre todo, al plano de la democracia; es decir, que se trabaje en proyectos mediante los cuales las escuelas se conviertan en espacios por excelencia para construir dinámicas democráticas, personas democráticas, grupos sociales y culturales democráticos, gobiernos democráticos; desde luego, teniendo claro que la escuela es tan sólo un ámbito dentro del marco de las instituciones que tienen responsabilidades políticas y sociales, pues "la democracia es una lucha no sólo pedagógica sino política y social".

Tener competencias sociales significa estar al tanto de lo que ocurre socialmente, la forma cómo ejercen el poder los grupos dominantes, lo que dicen, lo que callan, las formas culturales que imponen, las verdades que promueven y a las que les hacen eco, así como a la injusticia, con el objetivo de que, mediante acciones educativas y pedagógicas, se puedan conducir los análisis, las interpretaciones, las comprensiones hacia la construcción de sentido, que permita desvelar las estructuras de poder y emancipar la acción desde el conocimiento.

¿Qué se debe aprender en la escuela? Sobre esa pregunta debe interactuar todo intelectual transformativo; seguramente la respuesta no se puede reducir a conocimientos, normas y valores.

\section{Bibliografía}

Bravo Salinas, Néstor. Pedagogía problémica, Convenio Andrés Bello, Bogotá, 1997.

Canguilhem, George. "¿Qué es la psicología?". En: Revista Colombiana de Psicología No.7, Bogotá, Universidad Nacional de Colombia, 1998.

CarR, Wilfred y Kemmis, Stephen. Teoría crítica de la enseñanza, Barcelona, Martines Roca, 1988.

Consejo Nacional de Acreditación CNA, Decreto 272 de 1998, Bogotá.

DiAZ, Mario. "Pedagogía, discursoy poder". En: Pedagogía, Discurso y Poder, Bogotá, Corprodic, 1990.
Fuentes, Carlos. Prólogo a Educaciòn: la agenda para el siglo XXI, Programa de Naciones Unidas para el Desarrollo, Bogotá, TM Editores, director, Hernando Gómez Buendia, 1998.

Informe a la UNESCO sobre la educación para el siglo XXI, presidida por Jacques Delors, La educación encierra un tesoro. Madrid, Santillana, UNESCO, 1996.

KYmLickA, Hill. Ciudadanía multicultural, Barcelona, Paidós, 1995.

MARDONES, J. M. y otros. La ciencia crítica y la calidad educativa, Instituto de Ciencias de la Educación, Universidad del País Vasco.

GiRoux, Henry. Los profesores como intelectuales, hacia una pedagogía crítica del aprendizaje, Barcelona, Paidós, 1990.

Peña Rodríguez, Faustino. Pedagogía problémica: perspectivas curriculares y evaluativas, Módulo de pedagogía y currículo, Bogotá, Universidad Santo Tomás, 2001, mimeo.

ROLDÁn, Ofelia y otros. Educar, el desafío de hoy, Bogotá, Magisterio, 1999.

Tedesco, Juan Carlos. "Educación y sociedad en América Latina: algunos cambios conceptuales y políticos". En: Revista Colombiana de Educación No. 27, Bogotá, 1993.

Tetay, José María. Criterios para la construcción del PEI. Un Enfoque Investigativo, Bogotá, Magisterio, 1997.

TOURAINE, Alain, ¿Podremos vivir juntos? Fondo de Cultura Económica, Buenos Aires, 1997.

VASCO, Carlos Eduardo. Tres estilos de trabajo en las ciencias sociales, Bogotá, Cinep, 1990.

VATTIMO, Gianni. "Postmodernidad: ¿una sociedad transparente?". En: Colombia, el despertar de la modernidad, Foro Nacional por Colombia, Bogotá, 1991.

VILLAVECES, José Luis. "Racionalidad pública versus racionalidad privada: ¿ una falsa antinomia?". En: ¿Para dónde va Colombia?, compilación y análisis Hernando Gómez Buendia, Bogotá, Tercer MundoColciencias, 1999. 